\title{
Ambient air pollution and pulmonary vascular volume on computed tomography: the MESA Air Pollution and Lung cohort studies
}

\author{
Carrie P. Aaron ${ }^{1}$, Eric A. Hoffman², Steven M. Kawut ${ }^{3}$, John H.M. Austin ${ }^{4}$, \\ Matthew Budoff ${ }^{5}$, Erin D. Michos ${ }^{6}$, Karen Hinckley Stukovsky $^{7}$, Coralynn Sack ${ }^{8}$, \\ Adam A. Szpiro ${ }^{7}$, Karol D. Watson ${ }^{5}$, Joel D. Kaufman $\mathbb{1 0}^{8,9,10}$ and \\ R. Graham Barr ${ }^{1,11}$
}

@ERSpublications

In this large population-based study, higher ambient exposure to black carbon was associated with a greater volume of peripheral pulmonary vessels measured on noncontrast CT, suggesting diesel exhaust may impact small vessel remodelling in the lung http://ow.ly/nAEY30o8co4

Cite this article as: Aaron CP, Hoffman EA, Kawut SM, et al. Ambient air pollution and pulmonary vascular volume on computed tomography: the MESA Air Pollution and Lung cohort studies. Eur Respir J 2019; 53: 1802116 [https://doi.org/10.1183/13993003.02116-2018].

\begin{abstract}
Background: Air pollution alters small pulmonary vessels in animal models. We hypothesised that longterm ambient air pollution exposure would be associated with differences in pulmonary vascular volumes in a population-based study.

Methods: The Multi-Ethnic Study of Atherosclerosis recruited adults in six US cities. Personalised longterm exposures to ambient black carbon, nitrogen dioxide $\left(\mathrm{NO}_{2}\right)$, oxides of nitrogen $\left(\mathrm{NO}_{x}\right)$, particulate matter with a $50 \%$ cut-off aerodynamic diameter of $<2.5 \mu \mathrm{m}(\mathrm{PM} 2.5)$ and ozone were estimated using spatiotemporal models. In 2010-2012, total pulmonary vascular volume was measured as the volume of detectable pulmonary arteries and veins, including vessel walls and luminal blood volume, on noncontrast chest computed tomography (TPVVCT). Peripheral TPVVCT was limited to the peripheral $2 \mathrm{~cm}$ to isolate smaller vessels. Linear regression adjusted for demographics, anthropometrics, smoking, second-hand smoke, renal function and scanner manufacturer.

Results: The mean \pm SD age of the 3023 participants was $69.3 \pm 9.3$ years; $46 \%$ were never-smokers. Mean exposures were $0.80 \mu \mathrm{g} \cdot \mathrm{m}^{-3}$ black carbon, $14.6 \mathrm{ppb} \mathrm{NO}$ and $11.0 \mu \mathrm{g} \cdot \mathrm{m}^{-3}$ ambient PM2.5. Mean $\pm \mathrm{SD}$ peripheral TPVVCT was $79.2 \pm 18.2 \mathrm{~cm}^{3}$ and TPVVCT was $129.3 \pm 35.1 \mathrm{~cm}^{3}$. Greater black carbon exposure was associated with a larger peripheral TPVVCT, including after adjustment for city (mean difference 0.41 $(95 \%$ CI $0.03-0.79) \mathrm{cm}^{3}$ per interquartile range; $\left.\mathrm{p}=0.036\right)$. Associations for peripheral TPVVCT with $\mathrm{NO}_{2}$ were similar but nonsignificant after city adjustment, while those for PM2.5 were of similar magnitude but nonsignificant after full adjustment. There were no associations for $\mathrm{NO}_{x}$ or ozone, or between any pollutant and TPVVCT.

Conclusions: Long-term black carbon exposure was associated with a larger peripheral TPVVCT, suggesting diesel exhaust may contribute to remodelling of small pulmonary vessels in the general population.
\end{abstract}




\section{Introduction}

The pulmonary vasculature may be particularly sensitive to air pollution, as a significant portion of inhaled particulate matter and gaseous pollutants reach the richly vascularised alveolar bed $[1,2]$. In animal models, exposure to diesel exhaust and particulate matter with a 50\% cut-off aerodynamic diameter of $<2.5 \mu \mathrm{m}$ (PM2.5) increased right ventricular systolic pressure and resulted in muscularisation of pulmonary arterioles [3-5]. In humans, controlled exposure to diesel exhaust increased levels of endothelin-1, a pulmonary vasoconstrictor, and also increased pulmonary vascular resistance during dobutamine stress measured by transthoracic echocardiography [6-8]. Children with greater particulate matter exposures have also been found to have higher pulmonary arterial pressures on echocardiography [9].

Direct assessment of the pulmonary vasculature, by cardiac catheterisation or contrast-enhanced imaging, is challenging in the general population. In the Multi-Ethnic Study of Atherosclerosis (MESA) we found that higher long-term exposure to nitrogen dioxide $\left(\mathrm{NO}_{2}\right)$ and $\mathrm{PM} 2.5$ was associated with greater right ventricular mass on magnetic resonance imaging (MRI), suggesting chronically increased pulmonary arterial pressures $[10,11]$. However, to the best of our knowledge, no study has assessed the relationship of ambient air pollution and direct measures of pulmonary vascular structure in humans. We recently utilised a measure of the total pulmonary vascular volume assessed on noncontrast computed tomography (TPVVCT) [12], which we found to be increased in current and ex-smokers and reduced with airflow limitation [13]. We therefore hypothesised that in the general population exposure to black carbon, a marker of diesel exhaust, $\mathrm{PM} 2.5, \mathrm{NO}_{2}$, oxides of nitrogen $\left(\mathrm{NO}_{x}\right)$ and ozone would be associated with differences in TPVVCT in the whole lung and in the periphery of the lung, the latter chosen as a more sensitive marker of small pulmonary vessels.

\section{Methods}

\section{Multi-Ethnic Study of Atherosclerosis}

MESA is a prospective cohort study that recruited 6814 participants from six US cities who were aged 45-84 years and free of clinical cardiovascular disease in 2000-2002 [14]; 4716 participants ( $>70 \%$ of those still living) returned for follow-up in 2010-2012. The MESA Air Pollution Study (MESA Air) was a large ancillary study funded by the US Environmental Protection Agency (EPA) to add air pollution exposure assessments for consenting participants [15]. The MESA Lung Study enrolled 3965 MESA participants in 2004-2006 [16]; in 2010-2012 all were invited to undergo full-lung CT and spirometry, in addition to a random sample of those undergoing concurrent cardiac MRI (supplementary figure E1). This study includes 3023 participants who underwent lung CT and had at least one air pollutant estimate.

The protocols of MESA and all studies described herein were approved by the institutional review boards of all collaborating institutions and the US National Heart, Blood, and Lung Institute. All participants provided written informed consent.

\section{Pulmonary vascular volume on CT}

Noncontrast chest CT scans were acquired at suspended full inspiration on 64-detector scanners following the SPIROMICS protocol; reconstruction was performed in $0.625-0.75 \mathrm{~mm}$ increments using a high-spatial contrast algorithm [17]. All image analysis was performed at the MESA Lung CT Reading Center (University of Iowa, Iowa City, IA, USA) by trained and certified technicians without access to other participant information.

Dedicated software (Apollo; VIDA Diagnostics, Coralville, IA, USA) was used to segment the lungs and pulmonary vessels automatically with visual confirmation; vessels were identified using an approach previously described [12]. TPVVCT was measured as the volume of detectable arteries and veins, including vessel walls and luminal blood, down to $\sim 1 \mathrm{~mm}$ in diameter within segmented lung. The intraclass correlation coefficient on $10 \%$ replicate reads was 1.0. Peripheral TPVVCT was limited to the $2 \mathrm{~cm}$

Affiliations: 'Dept of Medicine, College of Physicians and Surgeons, Columbia University, New York, NY, USA. ${ }^{2}$ Dept of Radiology, University of lowa, lowa City, IA, USA. ${ }^{3}$ Depts of Medicine and Epidemiology, Perelman School of Medicine at the University of Pennsylvania, Philadelphia, PA, USA. ${ }^{4}$ Dept of Radiology, College of Physicians and Surgeons, Columbia University, New York, NY, USA. ${ }^{5}$ Dept of Medicine, University of California, Los Angeles, CA, USA. 'Dept of Medicine, Johns Hopkins University School of Medicine, Baltimore, MD, USA. ${ }^{7}$ Dept of Biostatistics, University of Washington, Seattle, WA, USA. ${ }^{8}$ Dept of Medicine, University of Washington, Seattle, WA, USA. ${ }^{9}$ Dept of Environmental and Occupational Health Sciences, University of Washington, Seattle, WA, USA. ${ }^{10}$ Dept of Epidemiology, University of Washington, Seattle, WA, USA. ${ }^{11}$ Dept of Epidemiology, Mailman School of Public Health, Columbia University, New York, NY, USA.

Correspondence: Carrie P. Aaron, Dept of Medicine, College of Physicians and Surgeons, Columbia University, PH 9 East Room 105, 630 West 168th Street, New York, NY 10032-3784, USA.

E-mail: cp2346@acolumbia.edu 
periphery of the lung excluding the portion of the lung adjacent to the mediastinum. Percent TPVVCT was indexed to CT lung volume.

Mean values of TPVVCT are $25-30 \%$ of average pulmonary blood volumes measured invasively [13]; this difference is not unexpected given the exclusion of large mediastinal pulmonary vessels and the inability to capture the microvasculature (vessels $<1 \mathrm{~mm}$ in diameter).

\section{Air pollution exposure estimates}

MESA Air generated cohort-specific prediction models of long-term exposure to ambient black carbon, $\mathrm{NO}_{2}, \mathrm{NO}_{x}, \mathrm{PM} 2.5$ and ozone. Estimates use maximum likelihood predictions with spatiotemporal modelling that have been previously described [18, 19]. Briefly, all models leverage measures from the MESA Air monitoring campaign in 2005-2009 that included two to five supplemental stationary monitors within each city and outdoor monitors at the homes of a subset of MESA participants. The models include all available measures from the EPA's Air Quality System monitors for all pollutants except for black carbon, due to different measurement techniques [20]. The models also include geographic variables such as land use (e.g. industrial, residential and water), distance to airports and coastlines, traffic patterns incorporated via dispersion models, population density, and urban topography $[18,20]$. Exposures were predicted in 2-week averages at each participant's home address [15] and estimates were weighted for time at each address if participants moved during the time of the exposure estimate.

Black carbon exposure was estimated using a spatial model from 2006-2008, the full years during which MESA Air monitoring occurred, while all other exposures were estimated as a 1-year average exposure prior to the participant's 2010-2012 visit. Figure 1 shows estimated exposure to black carbon in Chicago, IL. Black (or elemental) carbon estimates were based on the difference between pre- and post-exposure light absorption coefficients from reflectance on the Teflon filters used for PM2.5 monitoring (Smokestain Reflectometer 43D EEL; Diffusion Systems, London, UK) [20]. The relationship between black carbon concentration and light absorption was empirically determined in each city based on comparison to direct measurements [20].

\section{Covariate information}

Age, sex, race/ethnicity, educational attainment, income, pack-years of smoking, second-hand smoke exposure, medication use, medical history and smoking status were self-reported; current smoking was confirmed with urine cotinine $\left(>100 \mathrm{ng} \cdot \mathrm{mL}^{-1}\right)$ [16]. Height, weight, resting blood pressure, fasting serum glucose, creatinine, total cholesterol and high-density lipoproteins were measured using standard techniques [21]. Glomerular filtration rate was estimated using the Modification of Diet in Renal Disease equation [22]. Neighbourhood socioeconomic status (SES) index incorporates six US Census variables that are unique contributors to neighbourhood SES [23]. Hypertension was defined as blood pressure $\geqslant 140 / 90 \mathrm{mmHg}$ or self-reported hypertension and antihypertensive medication use. Diabetes was defined as fasting glucose $\geqslant 7.0 \mathrm{mmol} \cdot \mathrm{L}^{-1}\left(\geqslant 126 \mathrm{mg} \cdot \mathrm{dL}^{-1}\right)$, use of hypoglycaemic medication or self-reported physician diagnosis. Cardiac structure was assessed on cardiac-gated MRI on $1.5 \mathrm{~T}$ scanners with a phased-array surface coil [24]. Analysts blinded to participant information assessed left ventricular volumes and function using cine images of steady-state free precession pulse sequences acquired on two chambers, three chambers, four chambers and the short-axis plane using semiautomatic contouring (CIM version 6.0; UniServices, Auckland, New Zealand) [25].

Percent of emphysema-like lung ("percent emphysema") was defined as the percentage of voxels below $-950 \mathrm{HU}$ on full-lung CT scans obtained at coached full inspiration using dedicated software (Apollo). Spirometry was conducted in accordance with American Thoracic Society/European Respiratory Society guidelines [26]; all spirometry exams were reviewed by one investigator [27]. Airflow limitation was defined as pre-bronchodilator forced expiratory volume in $1 \mathrm{~s}(\mathrm{FEV} 1) /$ forced vital capacity $(\mathrm{FVC})<0.7$.

\section{Statistical analyses}

The sample was stratified by quintile of black carbon exposure for descriptive purposes. Linear regression was used to estimate the associations between air pollution exposure (independent variable) and TPVVCT (dependent variable), both treated as continuous, with sequentially adjusted models. The initial model adjusted for age, sex, race/ethnicity and height, to account for basic demographics and body size. The full model also adjusted for education, neighbourhood SES index (both related to SES), smoking status, pack-years, second-hand smoke exposure, renal function (to account for possible fluid retention), weight and scanner manufacturer (both accounting for scanner noise). The final model includes adjustment for city to address unmeasured confounding by study site. Missing data was minimal except for pack-years $(5 \%)$ and education $(<1 \%)$, and the issue was addressed by multiple imputation $(n=10)$. Generalised additive models were used to confirm linear associations between air pollutants and TPVVCT. In the 


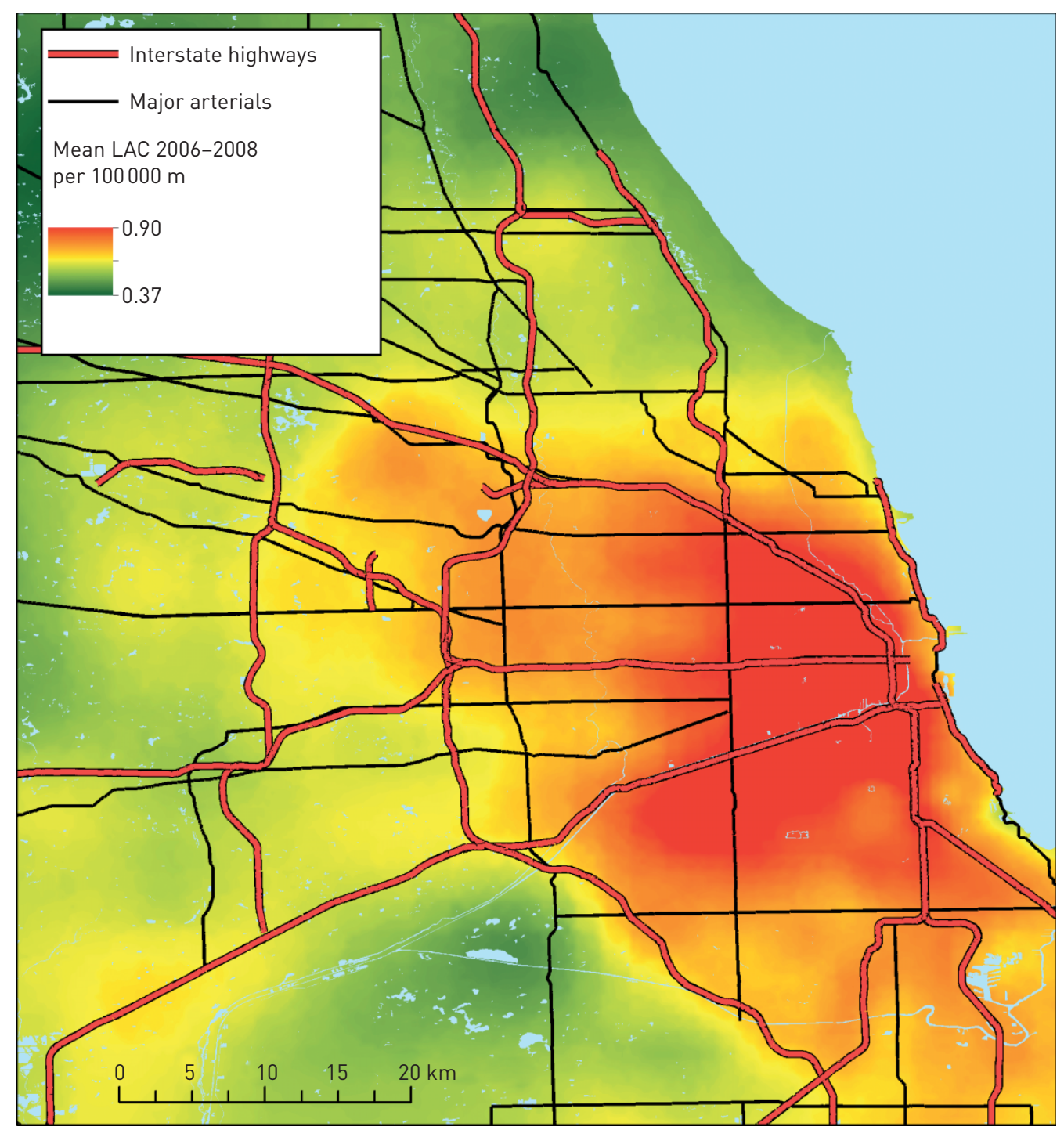

FIGURE 1 Map of estimated black carbon exposure derived from spatial averages over 2006-2008 in Chicago, IL. LAC: light absorption coefficient ( 0.5 per $100000 \mathrm{~m} \mathrm{LAC}$ is approximately equivalent to $0.5 \mu \mathrm{g} \cdot \mathrm{m}^{-3}$ black carbon).

analysis of black carbon and peripheral TPVVCT, effect modification was assessed on an additive scale using interaction terms by sex, race/ethnicity, age, smoking status, airflow limitation, CT manufacturer (all classified as shown in figure 3) and city.

Sensitivity analyses included additional adjustment for factors associated with pulmonary vascular volumes, including FEV1, percent emphysema, left ventricular end-diastolic volume and ejection fraction, diuretic use, and cardiac risk factors (hypertension, hyperlipidaemia and diabetes). We also assessed 5-year average exposures for $\mathrm{NO}_{2}, \mathrm{NO}_{x}$ and $\mathrm{PM} 2.5$ (to compare with the 2006-2008 black carbon estimates) and multipollutant models. We used pulmonary vascular volume (in millilitres) as the primary outcome in order to test associations with a single measure and also include results for percent TPVVCT, the ratio of pulmonary vascular volume to CT lung volume. Statistical significance was defined as a two-tailed p-value $<0.05$. Rather than adjust for multiple comparisons of these pollutants, which co-vary considerably, we report all analyses [28]. Analyses were performed using SAS version 9.4 (SAS Institute, Cary, NC, USA) and R (R Foundation for Statistical Computing, Vienna, Austria).

\section{Results}

\section{Study participants}

Of 4920 participants enrolled in the 2010-2012 MESA Exam, 3203 underwent full-lung CT scan and 3023 (94\%) had at least one air pollution estimate (supplementary figure E1). Included participants differed slightly from excluded participants in terms of demographics (supplementary table E1). Participants had a 
mean \pm SD age of $69 \pm 10$ years, 52\% female, 39\% White, 21\% Hispanic, 27\% African-American and $13 \%$ Chinese-American, and $46 \%$ were never-smokers. Mean \pm SD TPVVCT was $129.3 \pm 35.1 \mathrm{~cm}^{3}$ and peripheral TPVVCT was $79.2 \pm 18.2 \mathrm{~cm}^{3}$.

Mean \pm SD long-term exposures were $0.80 \pm 0.37 \mu \mathrm{g} \cdot \mathrm{m}^{-3}$ black carbon, $14.6 \pm 7.9 \mathrm{ppb} \mathrm{NO}_{2}, 26.0 \pm 16.3 \mathrm{ppb}$ $\mathrm{NO}_{x}, 11.0 \pm 1.5 \mu \mathrm{g} \cdot \mathrm{m}^{-3} \mathrm{PM} 2.5$ and $23.2 \pm 4.7 \mathrm{ppb}$ ozone, with an average inter-city interquartile range (IQR) of $0.15 \mu \mathrm{g} \cdot \mathrm{m}^{-3}, 4 \mathrm{ppb}, 8 \mathrm{ppb}, 1.3 \mu \mathrm{g} \cdot \mathrm{m}^{-3}$ and $3 \mathrm{ppb}$, respectively. Exposures varied by city, with the highest in New York, NY and Los Angeles, CA, and the lowest in St Paul, MN and Forsyth County, NC (supplementary figure E2). City-specific correlations for black carbon and $\mathrm{NO}_{2}$ were moderate to high (0.62-0.91), while those of black carbon and PM2.5 were variable (0.21-0.79) (supplementary table E2).

Compared with participants in the highest quintile of black carbon exposure, those in the lowest quintile were more likely to be White male ex-smokers with greater height, weight, educational attainment and second-hand smoke exposure, and were less likely to have diabetes and hypertension (table 1). Those in the lowest quintile had greater TPVVCT and peripheral TPVVCT, but lower percent TPVVCT and percent peripheral TPVVCT (normalised for lung volume), compared with the highest quintile.

\section{Ambient air pollution and pulmonary vascular volumes on CT}

A higher ambient black carbon exposure was associated with a greater peripheral TPVVCT after adjustment for age, sex, race/ethnicity and height, as well as in fully adjusted and city-adjusted models (city-adjusted mean difference 0.41 (95\% CI 0.03-0.79) $\mathrm{cm}^{3}$ per IQR; $\mathrm{p}=0.036$ ) (table 2). $\mathrm{NO}_{2}$ exposure was associated with a similar magnitude difference in peripheral TPVVCT in limited and fully adjusted models, but not after city adjustment. PM2.5 was associated with greater peripheral TPVVCT in the limited model and lost significance with full adjustment (table 2). There were no significant associations between $\mathrm{NO}_{x}$ or ozone exposure and peripheral TPVVCT (table 2). For comparison, an increase of 10 pack-years was associated with a $0.25 \mathrm{~cm}^{3}$ increase in peripheral TPVVCT in the city-adjusted model (95\% CI $\left.0.001-0.50 \mathrm{~cm}^{3} ; \mathrm{p}=0.045\right)$.

Higher PM2.5 exposure was associated with a greater TPVVCT in the limited model; however, the association was nonsignificant in fully adjusted and city-adjusted models. There were no significant associations between other pollutants and TPVVCT (table 2).

Given the more historical black carbon exposure estimates, we evaluated 5-year average exposures for other pollutants and results were unchanged (supplementary table E3). In multipollutant models including black carbon, $\mathrm{NO}_{2}$ and PM2.5 the difference in peripheral TPVVCT for an increase in black carbon remained of similar or greater magnitude, while the effect for other pollutants decreased (supplementary table E4). Generalised additive models for black carbon confirmed a linear association with peripheral TPVVCT across nearly all values of black carbon (figure 2).

Results for percent peripheral TPVVCT (scaled to CT lung volume) were significant for nearly all pollutants, with greater exposures associated with increased percent peripheral TPVVCT with the exception of ozone, which was in the opposite direction (supplementary table E5). Results for percent TPVVCT were very similar to those of percent peripheral TPVVCT (supplementary table E5). In addition, higher ozone exposure was associated with a larger CT lung volume in the full model ( $42 \mathrm{~mL}$ per IQR ozone; $\mathrm{p}<0.001$ ), whereas black carbon was not $(-8 \mathrm{~mL}$ per IQR black carbon; $\mathrm{p}=0.23)$.

While associations between black carbon exposure and peripheral TPVVCT were attenuated in ex-smokers and those with airflow limitation (figure 3), there was no significant effect modification, and results were similar across strata of sex, age, race/ethnicity and scanner manufacturer. Effect estimates for black carbon varied by city, with the strongest associations in New York, NY and Los Angeles, CA (supplementary figure E3), but there was not significant effect modification ( $p$-interaction $=0.42$ ). Results were unchanged with additional adjustment for $\mathrm{FEV} 1$, percent emphysema, left ventricular end-diastolic volume and ejection fraction, diuretic use and cardiac risk factors (figure 3).

\section{Discussion}

In this population-based study, higher black carbon exposure was associated with greater peripheral TPVVCT, a noninvasive CT measure of the pulmonary vasculature designed to isolate smaller vessels in the lung periphery. These findings were consistent after adjustment for multiple confounders including city, as well as in multipollutant models including $\mathrm{PM}_{2.5}$ and $\mathrm{NO}_{2}$, and suggest that long-term exposure to diesel exhaust may impact the pulmonary circulation, with a particular effect on smaller vessels. The magnitude of this change was comparable to the effect of $>15$ pack-years of smoking.

The pulmonary circulation is regulated by conditions within the lung and directly impacted by pulmonary disease. Within the lung, hypoxia causes localised pulmonary vasoconstriction [29], a response that optimises gas exchange but can be blunted in the setting of pulmonary infection [30, 31]. We have found 
TABLE 1 Characteristics of Multi-Ethnic Study of Atherosclerosis participants with total pulmonary vascular volume assessed on noncontrast computed tomography (TPVVCT) and air pollution estimates in 2010-2012 by quintile of black carbon exposure ${ }^{\#}$

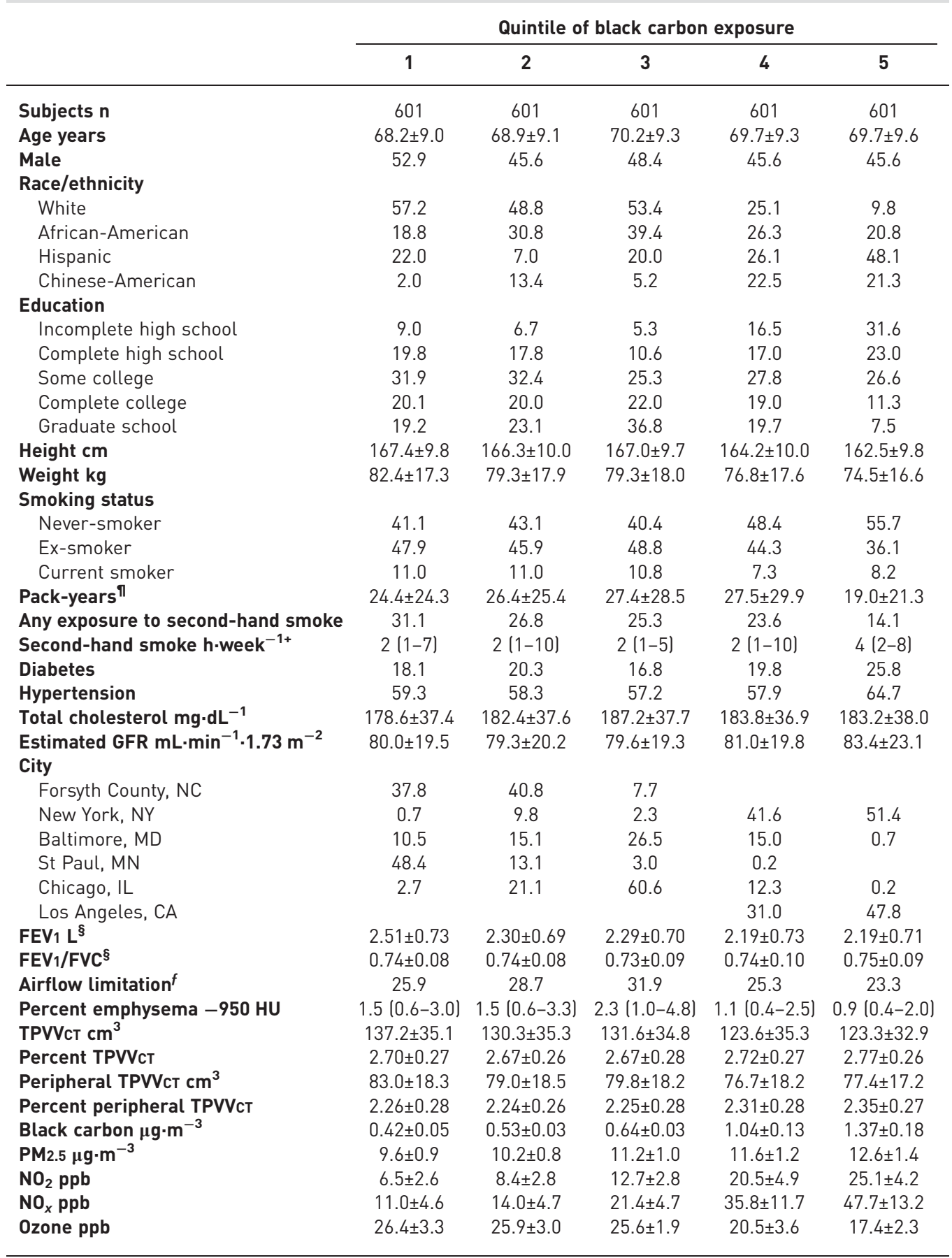

Data are presented as $\%$, mean \pm SD or median (interquartile range), unless otherwise stated. GFR: glomerular filtration rate; FEV1: forced expiratory volume in $1 \mathrm{~s} ; \mathrm{FVC}$ : forced vital capacity; PM2.5: particulate matter with a $50 \%$ cut-off aerodynamic diameter of $<2.5 \mu \mathrm{m} ; \mathrm{NO}_{2}$ : nitrogen dioxide; $\mathrm{NO}_{x}$ : oxides of nitrogen. ${ }^{\#}$ : $n=3005$ with measures of black carbon; ": among 1470 ever-smokers reporting pack-years; ${ }^{+}$: among 727 reporting any second-hand smoke exposure; ${ }^{\S}$ : among 2690 with grade A-D spirometry; $f$ : pre-bronchodilator FEV $1 / F V C<0.7$.

a lower pulmonary perfusion in those with chronic obstructive pulmonary disease and emphysema [32], and a higher TPVVCT in current and ex-smokers (with and without airflow limitation) [13]. In pulmonary hypertension, differences in pulmonary vascular volumes may depend on the cause; pulmonary vascular 
TABLE 2 Mean differences in total and peripheral pulmonary vascular volume associated with an interquartile range increase in long-term air pollutant exposure

\begin{tabular}{|c|c|c|c|c|}
\hline & $\begin{array}{l}\text { Mean difference in } \\
\text { TPVVct } \mathrm{cm}^{3}\end{array}$ & p-value & $\begin{array}{l}\text { Mean difference in } \\
\text { peripheral TPVVct } \mathrm{cm}^{3}\end{array}$ & p-value \\
\hline \multicolumn{5}{|l|}{$\begin{array}{l}\text { Black carbon, per } \\
0.15 \mu \mathrm{g} \cdot \mathrm{m}^{-3}(\mathrm{n}=3005)\end{array}$} \\
\hline $\begin{array}{l}\text { Age, sex, race and height } \\
\text { adjusted }\end{array}$ & $0.36(-0.01-0.73)$ & 0.06 & $0.30(0.09-0.50)$ & 0.005 \\
\hline Full model & $0.35(-0.03-0.73)$ & 0.07 & $0.31(0.10-0.53)$ & 0.004 \\
\hline Full model+city & $0.49(-0.19-1.18)$ & 0.16 & $0.41(0.03-0.79)$ & 0.036 \\
\hline \multicolumn{5}{|l|}{$\mathrm{NO}_{2}, \operatorname{per} 4 \mathrm{ppb}(\mathrm{n}=3005)$} \\
\hline $\begin{array}{l}\text { Age, sex, race and height } \\
\text { adjusted }\end{array}$ & $0.25(-0.21-0.70)$ & 0.29 & $0.27(0.02-0.53)$ & 0.035 \\
\hline Full model & $0.18(-0.28-0.64)$ & 0.44 & $0.27(0.01-0.53)$ & 0.040 \\
\hline Full model+city & $0.43(-0.41-1.28)$ & 0.32 & $0.40(-0.08-0.87)$ & 0.09 \\
\hline \multicolumn{5}{|l|}{$\mathrm{NO}_{x}$, per $8 \mathrm{ppb}(\mathrm{n}=3005)$} \\
\hline $\begin{array}{l}\text { Age, sex, race and height } \\
\text { adjusted }\end{array}$ & $0.15(-0.28-0.59)$ & 0.49 & $0.22(-0.02-0.47)$ & 0.07 \\
\hline Full model & $0.04(-0.42-0.49)$ & 0.88 & $0.19(-0.07-0.44)$ & 0.15 \\
\hline Full model+city & $0.25(-0.55-1.05)$ & 0.54 & $0.27(-0.18-0.73)$ & 0.20 \\
\hline \multicolumn{5}{|l|}{$\begin{array}{l}\text { PM2.5, per } 1.3 \mu \mathrm{g} \cdot \mathrm{m}^{-3} \\
(\mathrm{n}=3005)\end{array}$} \\
\hline $\begin{array}{l}\text { Age, sex, race and height } \\
\text { adjusted }\end{array}$ & $0.75(0.04-1.47)$ & 0.040 & $0.44(0.04-0.85)$ & 0.030 \\
\hline Full model & $0.46(-0.27-1.19)$ & 0.22 & $0.36(-0.06-0.77)$ & 0.09 \\
\hline Full model+city & $0.26(-0.64-1.16)$ & 0.57 & $0.21(-0.30-0.71)$ & 0.40 \\
\hline \multicolumn{5}{|l|}{ Ozone, per 3 ppb ( $n=3022)$} \\
\hline $\begin{array}{l}\text { Age, sex, race and height } \\
\text { adjusted }\end{array}$ & $-0.07(-0.66-0.51)$ & 0.81 & $-0.27(-0.60-0.05)$ & 0.10 \\
\hline Full model & $0.02(-0.58-0.61)$ & 0.96 & $-0.25(-0.59-0.08)$ & 0.14 \\
\hline Full model+city & $-0.23(-1.24-0.78)$ & 0.65 & $-0.18(-0.75-0.38)$ & 0.51 \\
\hline
\end{tabular}

Mean differences are presented with $95 \% \mathrm{Cl}$. TPVVст: total pulmonary vascular volume assessed on noncontrast computed tomography; $\mathrm{NO}_{2}$ : nitrogen dioxide; $\mathrm{NO}_{x}$ : oxides of nitrogen; $\mathrm{PM} 2.5$ : particulate matter with a $50 \%$ cut-off aerodynamic diameter of $<2.5 \mu \mathrm{m}$. Full model adjusts for age, sex, race/ethnicity, height, weight, education, neighbourhood socioeconomic status index, smoking status, pack-years, second-hand smoke exposure, estimated glomerular filtration rate and scanner manufacturer. Statistical significance was defined as a two-tailed $p$-value $<0.05$.

volumes are increased in pulmonary arterial hypertension [33] and decreased in chronic thromboembolic pulmonary hypertension [34]. Left heart dysfunction can also increase pulmonary pressures [35], although the overall effect on pulmonary vascular volume likely depends on the ability to maintain cardiac output.

A few prior studies have found associations between air pollution exposures and the pulmonary vasculature. This is the first study, to the best of our knowledge, to find an association between long-term ambient air pollution exposure and a direct measure of the pulmonary vasculature on CT. Prior pulmonary vascular assessments have been inferred by cardiac structure on MRI, with long-term $\mathrm{NO}_{2}$ and PM2.5 exposures associated with increased right ventricular mass $[10,11]$, or echocardiography, with $2 \mathrm{~h}$ of diesel exhaust exposure leading to increased pulmonary vascular resistance during exercise [8]; both suggest a link between air pollution and subclinical increases in pulmonary vascular pressures. In addition, a study of 81 children in Mexico found that those with greater long-term PM2.5 exposure had higher systolic pulmonary arterial pressure on echocardiography [9].

There are a few potential explanations for our findings of an increased volume of peripheral (smaller) pulmonary vessels with greater black carbon exposure. First, long-term ambient black carbon exposure may result in lung inflammation with increased parenchymal blood flow, as seen in the early stages of acute lung injury in animals [36-38]. A systemic inflammatory response occurs with chronic particulate matter exposure [39], suggesting an increase in pulmonary blood volume is possible. Second, the increased vascular volume may be due to smooth muscle hypertrophy in the vessel walls as seen in animals exposed to particulate matter and diesel exhaust [3-5], because the measure of TPVVCT includes the vessel walls as well as intraluminal blood volume. Third, black carbon exposure may contribute to vasoconstriction of the smallest pulmonary vessels (beyond the resolution of noncontrast CT), resulting in dilation of proximal 


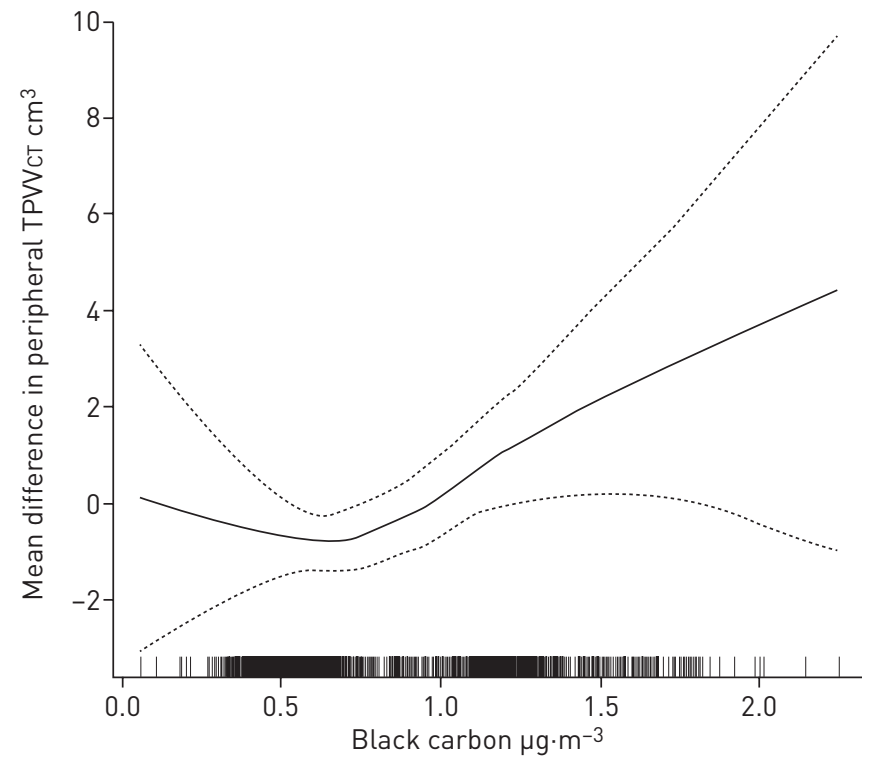

FIGURE 2 Generalised additive model showing the estimated mean difference in peripheral total pulmonary vascular volume assessed on noncontrast computed tomography (TPVVCT) over the range of black carbon exposure. Model adjusts for age, sex, race/ethnicity, height, weight, education, neighbourhood socioeconomic status index, smoking status, pack-years, second-hand smoke exposure, estimated glomerular filtration rate, scanner manufacturer and city. The dotted lines indicate $95 \% \mathrm{Cl}$. Each spectral line indicates one observation, showing the distribution of black carbon measurements.

upstream vessels. Unfortunately, we cannot distinguish between these mechanisms in the current study. Over time, any of these explanations may result in increased pulmonary vascular resistance [8], consistent with the increase in right ventricular mass seen with $\mathrm{NO}_{2}$ and $\mathrm{PM} 2.5$ exposure $[10,11]$.

While the differences observed in this general population sample are subclinical and not used clinically at the current time, they may be helpful in understanding how the pulmonary vasculature responds to therapy, which is in the initial phase of study in pulmonary hypertension and emphysema [40, 41]. Additionally, increases in pulmonary vascular volumes may reflect susceptibility to lung and vascular injury from air pollution exposure, with hypothesised implications for predicting the development of lung disease as well as other vascular diseases that have been linked to air pollution [42-44].

Significant city-specific results for black carbon were limited to New York, NY and Los Angeles, CA, where exposures were highest, although there was no effect modification by city. However, generalised additive models show a linear association across the range of exposures (figure 2). Results were also attenuated for those with airflow limitation and ex-smokers (groups which overlap considerably), despite the lack of effect modification. This may be related to a competing effect of airflow limitation and emphysema, diseases in which TPVVCT and small vessel volumes have been found to be decreased [13, 45]. Cigarette smoke itself may also increase pulmonary blood flow and angiogenesis, which may explain the weaker results in ex- and current smokers compared with never-smokers [46, 47].

Although we have previously found that ever-smokers with a lower TPVVCT have lower left ventricular end-diastolic volumes, suggesting that occult pulmonary vascular disease may be hard to distinguish from "diastolic dysfunction" [13], we do not suggest that either a high or low TPVVCT is better. Rather, these are all cross-sectional findings that may help us understand the impact of air pollution on the pulmonary vasculature and the interdependence of the heart and lungs.

In the current study, we found significant associations for peripheral TPVVCT, but not for TPVVCT. While this may be due to an isolated change in smaller vessels, as smaller vessels may be more sensitive to inhaled exposures, it is also possible that results for TPVVCT are confounded by body size and cardiac function. Body size more closely tracks with total lung volume than with peripheral $2 \mathrm{~cm}$ lung volume, which is the same $2 \mathrm{~cm}$ depth in all subjects, and after adjusting for CT lung volumes results were significant for both percent TPVVCT and percent peripheral TPVVCT. Cardiac function may impact vessels centrally more than peripherally, given the proximity to the heart, although results were not significantly changed after adjusting for left ventricular end-diastolic volume, ejection fraction and diuretic use.

We evaluated percent TPVVCT to further account for body size, and found significant results for black carbon for both percent TPVVCT and percent peripheral TPVVCT; however, results for ozone were 


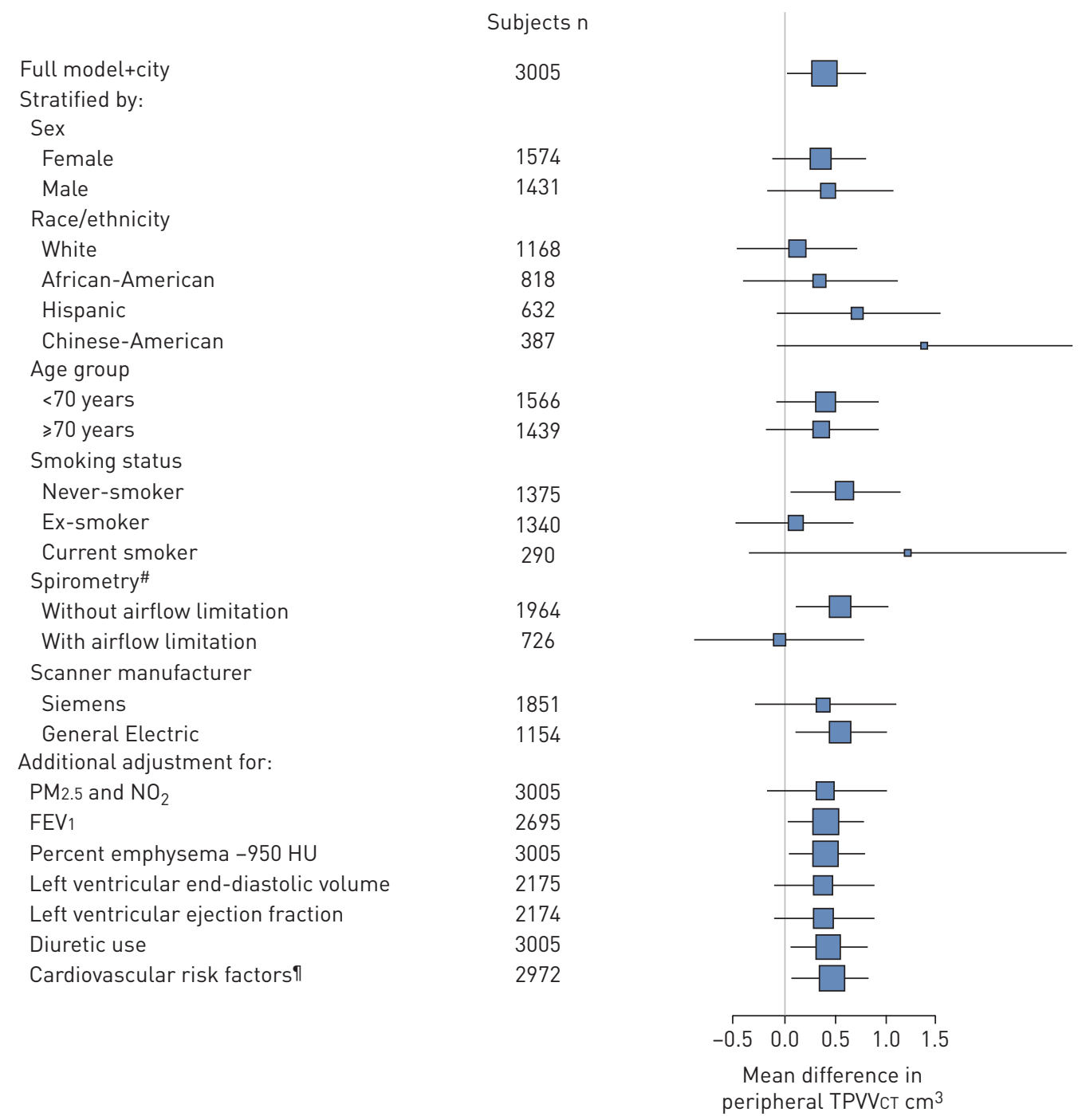

FIGURE 3 Sensitivity analyses showing estimated mean difference in peripheral total pulmonary vascular volume assessed on noncontrast computed tomography (TPVVCT) for an interquartile range increase in black carbon exposure. Results are adjusted for age, sex, race/ethnicity, height, weight, education, neighbourhood socioeconomic status index, smoking status, pack-years, second-hand smoke exposure, estimated glomerular filtration rate, scanner manufacturer and city. p-values for interactions: sex 0.46 , race/ethnicity 0.85 , age 0.19 , smoking status 0.84 , airflow limitation 0.74 and scanner manufacturer 0.99 . The size of the blue squares indicates the relative precision of the estimate, with larger squares reflecting more precision; $95 \% \mathrm{Cls}$ are indicated. PM2.5: particulate matter with a $50 \%$ cut-off aerodynamic diameter of $<2.5 \mu \mathrm{m} ; \mathrm{NO}_{2}$ : nitrogen dioxide: FEV1: forced expiratory volume in $1 \mathrm{~s} .{ }^{\#}$ : airflow limitation indicates pre-bronchodilator $\mathrm{FEV}_{1} /$ forced vital capacity $<0.7$ १: $^{\text {ๆ }}$ cardiovascular risk factors included total cholesterol, triglycerides, hypertension, systolic blood pressure, diabetes and glucose intolerance.

significant in the opposite direction. We found no evidence in our data that the negative association for ozone was due to confounding by lung function or the negative correlation between black carbon and ozone $(\mathrm{r}=-0.79)$. Instead, this finding likely relates to an association between higher ozone exposure and greater CT lung volume. Greater lung volumes have previously been reported in those living near major roadways [48]; however, the implications of this are not known.

\section{Limitations}

This is a large multiethnic study using state-of-the art air pollution estimates and novel imaging techniques; however, there are several limitations.

First, black carbon estimates are from 2006-2008, a more historical time-period than 1 year prior to full-lung CT scan used for other air pollutants due to availability of comparable historical data from the EPA. However, 5-year average exposures gave similar results to those for 1 year. 
Second, TPVVCT is not a measure of pulmonary arterial pressure and has not been validated against invasive measures, which were unfeasible in this large sample. Additionally, as the CT scans were without contrast, TPVVCT omits the microvasculature and does not separate arteries from veins. Thus, we are unable to determine if our findings reflect differences in the microvasculature, arteries or veins, or some combination thereof.

Third, selection bias, reverse causation and false-positive results should be considered in this cross-sectional study. MESA is a general population study that excluded those with clinical cardiovascular disease at baseline and good 10-year retention makes selection bias unlikely. Reverse causation is also unlikely with air pollution exposures. Although we performed multiple comparisons and results should be interpreted with caution, the consistent results for black carbon and suggestive results for $\mathrm{NO}_{2}$ make false-positive findings less likely.

\section{Conclusions}

In summary, we found that higher levels of black carbon exposure were associated with greater peripheral TPVVCT in this general population-based study, suggesting that long-term exposure to diesel exhaust may impact smaller peripheral lung vessels in the general population.

Acknowledgements: The authors thank the other investigators, staff and participants of the MESA (Multi-Ethnic Study of Atherosclerosis) study for their valuable contributions. A full list of participating MESA investigators and institutions can be found at www.mesa-nhlbi.org. The MESA study is conducted and supported by the US National Heart, Blood, and Lung Institute, National Institutes of Health and by the US Environmental Protection Agency (EPA) and MESA investigators. This publication was also developed under a STAR research assistance agreement, number RD831697 (MESA Air), awarded by the EPA. This publication has not been formally reviewed by the EPA. The views expressed in this document are solely those of the authors and the EPA does not endorse any products or commercial services mentioned in this publication.

Conflict of interest: C.P. Aaron reports grants from NIH, during the conduct of the study; grants from Alphal Foundation and Stony Wold-Herbert Fund, outside the submitted work. E.A. Hoffman reports grants from NIH, during the conduct of the study; grants from NIH, outside the submitted work; and is a founder and shareholder of VIDA Diagnostics, from whom software was utilised for the data analysis. S.M. Kawut reports grants from NIH, during the conduct of the study; grants from NIH, Actelion, United Therapeutic, Gilead, Lung Biotech, Pfizer, Ikaria, Merck, Bayer, Pulmonary Hypertension Association and GeNO, travel reimbursement from American College of Chest Physicians and American Thoracic Society, and personal fees from European Respiratory Journal, outside the submitted work. J.H.M. Austin reports grants from NIH, during the conduct of the study; personal fees from PulmonX, outside the submitted work. M. Budoff reports grants from NIH, during the conduct of the study; grants from GE, outside the submitted work. E.D. Michos reports personal fees from Siemens Healthcare Diagnostics, outside the submitted work. K. Hinckley Stukovsky reports salary support from Cystic Fibrosis Foundation, outside the submitted work. C. Sack has nothing to disclose. A.A. Szpiro has nothing to disclose. K.D. Watson reports grants from NIH, during the conduct of the study. J.D. Kaufman reports grants from US EPA, during the conduct of the study. R.G. Barr reports grants from NIH, during the conduct of the study; grants from NIH, Alphal Foundation and COPD Foundation, outside the submitted work.

Support statement: NIH/National Heart, Blood, and Lung Institute R01-HL077612, R01-HL093081, N01-HC95159, N01-HC95160, N01-HC95161, N01-HC95162, N01-HC95163, N01-HC95164, N01-HC95165, N01-HC95169, UL1TR000040; US Environmental Protection Agency R831697. Funding information for this article has been deposited with the Crossref Funder Registry.

\section{References}

1 Lippman M, Leikauf GD. Introduction and background. In: Lippman M, ed. Environmental Toxicants. 3rd Edn. New York, Wiley, 2009; pp. 1-38.

2 Weibel ER. Morphometry of the Human Lung. Berlin, Springer, 1963.

3 Liu J, Ye X, Ji D, et al. Diesel exhaust inhalation exposure induces pulmonary arterial hypertension in mice. Environ Pollut 2018; 237: 747-755.

4 Lemos M, Mohallen SV, Macchione $\mathrm{M}$, et al. Chronic exposure to urban air pollution induces structural alterations in murine pulmonary and coronary arteries. Inhal Toxicol 2006; 18: 247-253.

5 Rivero DH, Soares SR, Lorenzi-Filho G, et al. Acute cardiopulmonary alterations induced by fine particulate matter of Sao Paulo, Brazil. Toxicol Sci 2005; 85: 898-905.

6 Lund AK, Lucero J, Lucas S, et al. Vehicular emissions induce vascular MMP-9 expression and activity associated with endothelin-1-mediated pathways. Arterioscler Thromb Vasc Biol 2009; 29: 511-517.

7 Peretz A, Sullivan JH, Leotta DF, et al. Diesel exhaust inhalation elicits acute vasoconstriction in vivo. Environ Health Perspect 2008; 116: 937-942.

8 Wauters A, Vicenzi M, De Becker B, et al. At high cardiac output, diesel exhaust exposure increases pulmonary vascular resistance and decreases distensibility of pulmonary resistive vessels. Am J Physiol Heart Circ Physiol 2015; 309: H2137-H2144.

9 Calderon-Garciduenas L, Vincent R, Mora-Tiscareno A, et al. Elevated plasma endothelin-1 and pulmonary arterial pressure in children exposed to air pollution. Environ Health Perspect 2007; 115: 1248-1253.

10 Leary PJ, Kaufman JD, Barr RG, et al. Traffic-related air pollution and the right ventricle. The Multi-Ethnic Study of Atherosclerosis. Am J Respir Crit Care Med 2014; 189: 1093-1100.

11 Aaron CP, Chervona Y, Kawut SM, et al. Particulate matter exposure and cardiopulmonary differences in the Multi-Ethnic Study of Atherosclerosis. Environ Health Perspect 2016; 124: 1166-1173. 
12 Shikata H, McLennan G, Hoffman EA, et al. Segmentation of pulmonary vascular trees from thoracic 3D CT images. Int J Biomed Imaging 2009; 2009: 636240.

13 Aaron CP, Hoffman EA, Lima JAC, et al. Pulmonary vascular volume, impaired left ventricular filling and dyspnea: the MESA Lung Study. PLoS One 2017; 12: e0176180.

14 Bild DE, Bluemke DA, Burke GL, et al. Multi-Ethnic Study of Atherosclerosis: objectives and design. Am J Epidemiol 2002; 156: 871-881.

15 Kaufman JD, Adar SD, Allen RW, et al. Prospective study of particulate air pollution exposures, subclinical atherosclerosis, and clinical cardiovascular disease: the Multi-Ethnic Study of Atherosclerosis and Air Pollution (MESA Air). Am J Epidemiol 2012; 176: 825-837.

16 Rodriguez J, Jiang R, Johnson WC, et al. The association of pipe and cigar use with cotinine levels, lung function, and airflow obstruction: a cross-sectional study. Ann Intern Med 2010; 152: 201-210.

17 Sieren JP, Newell JD Jr, Barr RG, et al. SPIROMICS protocol for multicenter quantitative CT to phenotype the lungs. Am J Respir Crit Care Med 2016; 194: 794-806.

18 Keller JP, Olives C, Kim SY, et al. A unified spatiotemporal modeling approach for predicting concentrations of multiple air pollutants in the Multi-Ethnic Study of Atherosclerosis and Air Pollution. Environ Health Perspect 2015; 123: 301-309.

19 Sampson PD, Szpiro AA, Sheppard L, et al. Pragmatic estimation of a spatio-temporal air quality model with irregular monitoring data. Atmos Environ 2011; 45: 6593-6606.

20 Cohen MA, Adar SD, Allen RW, et al. Approach to estimating participant pollutant exposures in the Multi-Ethnic Study of Atherosclerosis and Air Pollution (MESA Air). Environ Sci Technol 2009; 43: 4687-4693.

21 MESA. MESA Exam 5: Field Center Procedures: Manual of Operations. 2010. www.mesa-nhlbi.org/PublicDocs/ 2011/MESAE5_MOPJanuary2011.pdf Date last accessed: March 24, 2019.

22 Levey AS, Bosch JP, Lewis JB, et al. A more accurate method to estimate glomerular filtration rate from serum creatinine: a new prediction equation. Modification of Diet in Renal Disease Study Group. Ann Intern Med 1999; 130: 461-470.

23 Hajat A, Diez-Roux AV, Adar SD, et al. Air pollution and individual and neighborhood socioeconomic status: evidence from the Multi-Ethnic Study of Atherosclerosis (MESA). Environ Health Perspect 2013; 121: $1325-1333$.

24 Liu CY, Liu YC, Wu C, et al. Evaluation of age-related interstitial myocardial fibrosis with cardiac magnetic resonance contrast-enhanced $\mathrm{T}_{1}$ mapping: MESA (Multi-Ethnic Study of Atherosclerosis). J Am Coll Cardiol 2013; 62: $1280-1287$.

25 Donekal S, Venkatesh BA, Liu YC, et al. Interstitial fibrosis, left ventricular remodeling, and myocardial mechanical behavior in a population-based multiethnic cohort: the Multi-Ethnic Study of Atherosclerosis (MESA) study. Circ Cardiovasc Imaging 2014; 7: 292-302.

26 Miller MR, Hankinson J, Brusasco V, et al. Standardisation of spirometry. Eur Respir J 2005; 26: 319-338.

27 Hankinson JL, Kawut SM, Shahar E, et al. Performance of American Thoracic Society-recommended spirometry reference values in a multiethnic sample of adults: the Multi-Ethnic Study of Atherosclerosis (MESA) Lung Study. Chest 2010; 137: 138-145.

28 Rothman KJ. No adjustments are needed for multiple comparisons. Epidemiology 1990; 1: 43-46.

29 Hales CA. The site and mechanism of oxygen sensing for the pulmonary vessels. Chest 1985; 88: Suppl., 235S-240S.

30 Hoffman EA, Simon BA, McLennan G. A structural and functional assessment of the lung via multidetector-row computed tomography: phenotyping chronic obstructive pulmonary disease. Proc Am Thorac Soc 2006; 3: 519-532.

31 Gust R, Kozlowski J, Stephenson AH, et al. Synergistic hemodynamic effects of low-dose endotoxin and acute lung injury. Am J Respir Crit Care Med 1988; 157: 1919-1926.

32 Hueper K, Vogel-Claussen J, Parikh MA, et al. Pulmonary microvascular blood flow in mild chronic obstructive pulmonary disease and emphysema. The MESA COPD Study. Am J Respir Crit Care Med 2015; 192: 570-580.

33 Rengier F, Worz S, Melzig C, et al. Automated 3D volumetry of the pulmonary arteries based on magnetic resonance angiography has potential for predicting pulmonary hypertension. PLoS One 2016; 11: e0162516.

34 Derlin T, Kelting C, Hueper K, et al. Quantitation of perfused lung volume using hybrid SPECT/CT allows refining the assessment of lung perfusion and estimating disease extent in chronic thromboembolic pulmonary hypertension. Clin Nucl Med 2018; 43: e170-e177.

35 Gerges M, Gerges C, Lang IM. How to define pulmonary hypertension due to left heart disease. Eur Respir J 2016; 48: 553-555.

36 Richter T, Bergmann R, Knels L, et al. Pulmonary blood flow increases in damaged regions directly after acid aspiration in rats. Anesthesiology 2013; 119: 890-900.

37 Richter T, Bergmann R, Pietzsch J, et al. Effects of pulmonary acid aspiration on the regional pulmonary blood flow within the first hour after injury: an observational study in rats. Clin Hemorheol Microcirc 2015; 60: 253-262.

38 Richter T, Bergmann R, Musch G, et al. Reduced pulmonary blood flow in regions of injury 2 hours after acid aspiration in rats. BMC Anesthesiol 2015; 15: 36.

39 van Eeden SF, Yeung A, Quinlam K, et al. Systemic response to ambient particulate matter: relevance to chronic obstructive pulmonary disease. Proc Am Thorac Soc 2005; 21: 61-67.

40 Skrok J, Shehata ML, Mathai S, et al. Pulmonary arterial hypertension: MR imaging-derived first-pass bolus kinetic parameters are biomarkers for pulmonary hemodynamics, cardiac function, and ventricular remodeling. Radiology 2012; 263: 678-687.

41 Iyer KS, Newell JD Jr, Jin D, et al. Quantitative dual-energy computed tomography supports a vascular etiology of smoking-induced inflammatory lung disease. Am J Respir Crit Care Med 2016; 193: 652-661.

42 Kaufman JD, Adar SD, Barr RG, et al. Association between air pollution and coronary artery calcification within six metropolitan areas in the USA (the Multi-Ethnic Study of Atherosclerosis and Air Pollution): a longitudinal cohort study. Lancet 2016; 388: 696-704.

43 Parker JD, Kravets N, Vaidyanathan A. Particulate matter air pollution exposure and heart disease mortality risks by race and ethnicity in the United States: 1997-2009 NHIS with mortality followup through 2011. Circulation 2018; 137: 1688-1697. 
44 Chen H, Burnett RT, Kwong JC, et al. Spatial association between ambient fine particulate matter and incident hypertension. Circulation 2014; 129: 562-569.

45 Estepar RS, Kinney GL, Black-Shinn JL, et al. Computed tomographic measures of pulmonary vascular morphology in smokers and their clinical implications. Am J Respir Crit Care Med 2013; 188: 231-239.

46 Gilman MJ, Sylvester JT, Kennedy TP, et al. Vascular effects of cigarette smoke in isolated pig lungs. Am Rev Respir Dis 1981; 124: 549-553.

47 Heeschen C, Jang JJ, Weis $\mathrm{M}$, et al. Nicotine stimulates angiogenesis and promotes tumor growth and atherosclerosis. Nat Med 2001; 7: 833-839.

48 Rice MB, Li W, Dorans KS, et al. Exposure to traffic emissions and fine particulate matter and computed tomography measures of the lung and airways. Epidemiology 2018; 29: 333-341. 